\title{
Isolation of Zebrafish gdf7 and Comparative Genetic Mapping of Genes Belonging to the Growth/Differentiation Factor 5, 6, 7 Subgroup of the TGF- $\beta$ Superfamily
}

\author{
Alan J. Davidson, ${ }^{1}$ John H. Postlethwait, ${ }^{2}$ Yi-Lin Yan, ${ }^{2}$ David R. Beier, ${ }^{3}$ \\ Cherie van Doren, ${ }^{3}$ Dorothee Foernzler, ${ }^{3}$ Anthony J. Celeste, ${ }^{4}$ Kathryn E. Crosier, ${ }^{1}$ \\ and Philip S. Crosier ${ }^{1,5}$ \\ ${ }^{1}$ Department of Molecular Medicine, School of Medicine, University of Auckland, Auckland, New Zealand; ${ }^{2}$ Institute \\ of Neuroscience, University of Oregon, Eugene, Oregon 97403-1254 USA; ${ }^{3}$ Brigham and Women's Hospital Harvard \\ Medical School, Boston, Massachusetts 02115 USA; ${ }^{4}$ Genetics Institute, Cambridge, Massachusetts 02140 USA
}

\begin{abstract}
The Growth/differentiation factor (Gdf) 5, 6, 7 genes form a closely related subgroup belonging to the TGF- $\beta$ superfamily. In zebrafish, there are three genes that belong to the Gdf5, 6, 7 subgroup that have been named radar, dynamo, and contact. The genes radar and dynamo both encode proteins most similar to mouse GDF6. The orthologous identity of these genes on the basis of amino acid similarities has not been clear. We have identified gdf7, a fourth zebrafish gene belonging to the Gdf5, 6, 7 subgroup. To assign correct orthologies and to investigate the evolutionary relationships of the human, mouse, and zebrafish Gdf5, 6, 7 subgroup, we have compared genetic map positions of the zebrafish and mammalian genes. We have mapped zebrafish gdf7 to linkage group (LG) 17, contact to LG9, GDF6 to human chromosome (Hsa) 8 and GDF7 to Hsa2p. The radar and dynamo genes have been localized previously to LG16 and LG19, respectively. A comparison of syntenies shared among human, mouse, and zebrafish genomes indicates that gdf7 is the ortholog of mammalian GDF7/Gdf7. LG16 shares syntenic relationships with mouse chromosome (Mmu) 4, including Gdf6. Portions of LG16 and LG19 appear to be duplicate chromosomes, thus suggesting that radar and dynamo are both orthologs of Gdf6. Finally, the mapping data is consistent with contact being the zebrafish ortholog of mammalian GDF5/Gdf5.
\end{abstract}

[The sequence data described in this paper have been submitted to the GenBank data library under accession numbers AF113022 and AF113023.]

The TGF- $\beta$ superfamily is a large group of genes encoding secreted signaling molecules that regulate a diverse range of biological processes during growth, repair, and embryonic development (Kingsley 1994; Hogan 1996). TGF- $\beta$-related peptides are synthesized as large precursor molecules comprised of these two major domains: a poorly conserved amino-terminal preprodomain and a highly conserved carboxy-terminal mature domain. For most superfamily members, the mature domain contains seven invariant cysteine residues that are involved in intramolecular and intermolecular disulphide bonds (Daopin et al. 1992; Schlunegger and Grütter 1992; Griffith et al. 1996; Eigenbrot and Gerber 1997). The active signaling molecule is a homo- or heterodimer of the mature domain that is released from the prodomain by cleavage at a dibasic R-X-X-R site (in which X is any amino acid; Dubois et al. 1995; Nachtigal and Ingraham 1996).

Members of the TGF- $\beta$ superfamily can be orga-

${ }^{5}$ Corresponding author.

E-MAIL ps.crosier@auckland.ac.nz; FAX (649) 373-7492. nized into related subgroups on the basis of amino acid similarity within the mature domain. The mouse Growth/differentiation factor (Gdf) 5, 6, and 7 genes were originally isolated from genomic DNA by a degenerate polymerase chain reaction approach and homologous genes have been identified in other mammals and zebrafish (Chang et al. 1994; Storm et al. 1994; Rissi et al. 1995; Bruneau and Rosa 1997; Bruneau et al. 1997; Wolfman et al. 1997). In the mouse and human, these genes show distinct patterns of expression in developing cartilage and joints (Chang et al. 1994; Storm et al. 1994; Storm and Kingsley 1996; Wolfman et al. 1997). Gdf5 was mapped to a region of mouse chromosome 2 that contains the brachypodism mutation. Mutations in Gdf5 were found to be responsible for the brachypodism phenotype (Storm et al. 1994), whereas mutations in the human ortholog (also known as CDMP1) cause the phenotypically similar human disorder HunterThompson type chondrodysplasia (Thomas et al. 1996). In addition to their role in connective tissue development, GDF5, 6, and 7 also have effects on other 
tissues. A targeted mutation in the mouse $G d f 7$ gene results in hydrocephalus and a defect in the development of discrete dorsal commissural neurons (Lee et al. 1998). GDF6 and GDF7 inhibit terminal differentiation of myoblasts (Inada et al. 1996), whereas GDF5 can act as a neurotrophic and angiogenic factor (Krieglstein et al. 1995; Yamashita et al. 1997).

In zebrafish, three genes belonging to the $G d f 5,6$, 7 subgroup have been reported and named radar, $d y$ namo, and contact (Rissi et al. 1995; Bruneau and Rosa 1997; Bruneau et al. 1997). Multiple tissues express radar during embryonic development, including putative neural crest cells, the neural tube, and the retina (Rissi et al. 1995). Expression of dynamo is found in posterior neural tissue during the development of the central nervous system (Bruneau and Rosa 1997), whereas contact is expressed in the pectoral fin buds and the developing cartilage of the head (Bruneau et al. 1997). The orthologous assignment of radar, dynamo, contact, and their evolutionary relationship to mammalian Gdf5, 6, 7 genes has been unclear.

In this report, we describe the isolation of zebrafish $g d f 7$, a fourth gene belonging to the Gdf5, 6, 7 subgroup. The radar and dynamo genes have been localized to LG16 and LG19 (Postlethwait et al. 1998). We have mapped genetically the remaining zebrafish genes $g d f 7$ and contact, as well as human GDF6 and GDF7. A comparison of syntenies shared among human, mouse, and zebrafish genomes suggests that $g d f 7$ is the ortholog of mammalian $G D F 7 / G d f 7$. Both radar and $d y$ namo appear to be orthologs of murine Gdf6 that have arisen from a chromosomal duplication event that has occured in teleosts. Finally, the mapping data is consistent with contact being the zebrafish ortholog of GDF5/Gdf5.

\section{RESULTS}

Isolation of $g d f 7$

and Phylogenetic Analysis of the Gdf5, 6, 7 Subgroup

A genomic library was screened with a probe encoding the mature domain of murine GDF7. AF113023.
Twenty of the strongest positive plaques were further purified, subcloned, and sequenced. In addition to the previously published genes radar, dynamo, and contact (Rissi et al. 1995; Bruneau and Rosa 1997; Bruneau et al. 1997), a fourth gene was identified that we have named $g d f 7$. Although a $g d f 7$ cDNA failed to be isolated from two independent cDNA libraries, the ORF identified in the $g d f 7$ genomic fragment was extended by an additional 137 amino acids by the technique of 5' rapid amplification of cDNA ends (5' RACE) on cDNA derived from adult testis tissue. The combined nucleotide sequence and conceptual translation of $g d f 7$ is shown in Figure 1. An analysis of the $g d f 7$ ORF failed to identify a potential start methionine and hydrophobic
ATCGGCGCTGTATCCCTCTGATAACGGGCTCCACTCACATCTGGATGCGCGCGGTCCAGCAGGAACCAGCGCGAGCTC

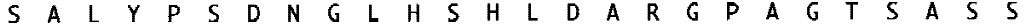
CCGGAACGAGT CCACATCTGTTCATGTTCCGTCTTACATGATCTCTCIGTACAGAACTCTGTCTGAACTGGACCGACG $\begin{array}{llllllllllllllllllllllllll}R & N & E & S & T & S & V & H & V & P & S & Y & M & I & S & L & Y & R & T & L & S & E & L & D & R & R\end{array}$ GACTGGTAACGGCAGTCACACGCGCTCCAGGAGATATGCCAACACGGTCACCAGCTTCATGGATCTGGGGCAAGATGA

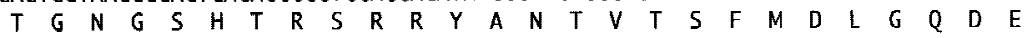
GCCTTCACTTATGTTCCAGCAGCAGTACACATTCAACCTGTCTGGACTCTCCCGACTGGATGAACTGATGGAAGTGGA

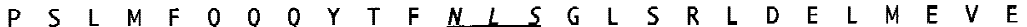
GCTGCGGGTTCTGAGGAGGCCACCGCAAGATGTCTTAAGCTTACTCTCTTATGGTGGGAACCTGTATCGTCTGCTCCT $\begin{array}{llllllllllllllllllllllllll}L & R & V & L & R & R & P & P & Q & D & V & L & S & L & L & S & Y & G & G & N & L & Y & R & L & L & L\end{array}$ TCACACCTGCTCACTTCCAGGATCTCTTCACCAACCTTTGCTTCTCAGCTCCAGGACCATCGATCTTCTAGATCTTCT

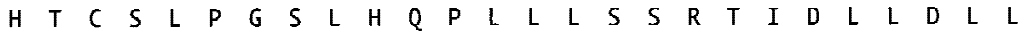
AGATACCTCTTCAGCCACATGGGATGTGTTTGATGTGGGCCCAATCATAAAGACCCCCCTCAAGCAGCACAGGACTGC

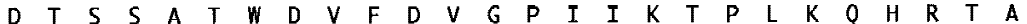
AGAAGACACTAGGTTGTTGTGCCTTAGCATATCTGCTGTTTCTGATTCAAATAACGAGGCTGTGCATCCTGGGATGCT

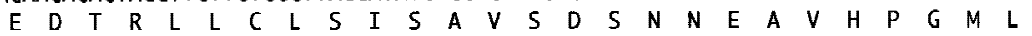
GGGTCTAAGCCGCGAGGATCAGCAGACCCATGAAAGAGCCTTACTAGTGGCGITTTCTCAAGCACGGAGGAAGGAGAA $\begin{array}{llllllllllllllllllllllllll}G & L & S & R & E & D & Q & Q & T & H & E & R & A & L & L & V & A & F & S & Q & A & R & R & K & E & N\end{array}$ CCTCTTTAGGGAGATCCGTGAGAAGATAAGGGCTATGAAAAGTCGGAAGTTTTCTAATCCCACACCAGAGCATAGTAT

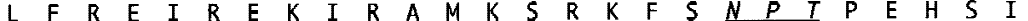
CAAAGGTCATCCGAGGCATCGGCGGAGGAGAAGGACAGCACTAGCAGGCCGTCCCGGAGTAGGACCCATTACCAGCGG $\begin{array}{lllllllllllllllllllllllllll}K & G & H & P & R & H & R & R & R & R & R & T & A & L & A & G & R & P & G & V & G & P & I & T & S & G\end{array}$ AGGAAAGGGAGGTGGAAGACGGAGGACACGCTGCAGCCGGAAACCGCTTCACGTGAATTTCAAAGAACTTGGTTGGGA $\begin{array}{llllllllllllllllllllllllll}G & K & G & G & G & R & R & R & T & R & (C) & S & R & K & P & L & H & V & N & F & K & E & L & G & W & D\end{array}$ TGACTGGATTATTGCACCGCTGGATTATGAAGCATATCACTGTGAGGGTTTGTGTGACTTCCCTTTGCGCTCACACCT D W I I A A P $L$ L D GGAACCAACCAACCATGCCATTATTCAGACCCTCATGAACTCCATGGACCCAGAGTCCACCCCTCCCAGTTGTTGTGT

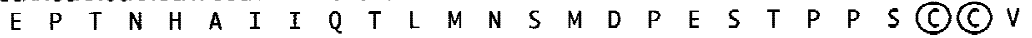
ACCCTCAAAACTCAGTCCCATCAGCATTCTGTACATTGACTCGGGGAATAATGTGGTTTATAAACAGTACGAGGACAT

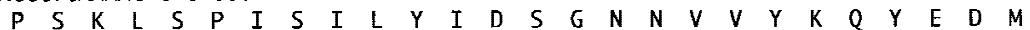
GGTGGTGGAGAGTTGTGGGTGCAGGTAGCAATGTGAGCAAGGGGTGAGCGACCCCTGGACACTACCCTAAAGCCTCAT $V$ V E S (C) G (C) R .

TCGCGGAGGCTGAGCAAAGCATTGTGGGCAAGACGAAGCAGTCGGAGCTGGTGTAAATGTGTGACAACGAGCATGTGT GTATGTTCAGATGGCGATAAGACAAT GAGTTGTTTTTTTAGAAGCGAGCGTAGGACATGTGTGCCGAGCAGCTCTAAAT TCCCCTTACAACACCAAATAAACATCTCCTCAACGGAAAAATGAGTGAAAACCTGGGCTTTCCAGAAATTATTTTACA TCCTGTTAATGTAAACTCAGTGATAGAGTGTTGAAGTATCCTTGCAGCAGCGATAGAAATGTTGGCCCTGTTAAACTC TGTCAGAGTCTGGGAATCTGTCAGCGGCAGCACACAACTATAACTTGTATAACAGTAAAGGTGCTTTGCATGACAGTC AGCTTATAGTCAAGAAGAAATAGCTCAGAAGAGTTTGTAATGCCTCTCTGAGGAGTCTAGCGGTCCTAATCAGATATG GCATTGACTGAGCACTTTTCACTCCAAAGTTTTCCTGGTTAGCATGGCTTCGTTAGCCCCTGTTGGAAGATGTTGTAA CTACAACATGTTGTAACCACCATGGCTCTCAGTTGAATTCTATTCAAAATAGCACATTTTTTATGAGTCAACTGATTGG CAAGAAAACTGGGTGCAGGAATCAGCACTCAAGAACAAATCTGAGAGATTTGAAAACATAATACTCACATAATACTCA CCAATAGCTTTCTTAGTCCTCCATGCAGTATGTTTTTGAAGGCTTTAGAGGCTTTTAGAATTATAAAGGTAGACAACTTC CAATCTCTAATGTTCAAAAGTTCATATCAAGCTTCCTAAAACTTCACAAGGGTTGTTTTAGTTCATCATTTTATATTCT TAAAATAACGTTTTGAATGCTAAAATTAAGCTGCTTAACGAGAAAGAGAAAGGCGTTTTIATGAAAAATAAAATGATGA TGACACCACATTTGGCTTGGGTCAGATGATAAAGCTGCAGTATAGTACATGATGTGTGCAATATGTGAAAGACAAATG ACTTCATTGCATCGAGTGACCTCAGACCTTATTTTGTGAAAGGCGCAGTGAAGCCAAAATCAAAATGATTGCTTTGTT TTATCTCGACACCAGATTTGGAGATCTACTTTCAGCGTTCACCTACATTGACTGAGAGGGTGTG

Figure 1 Nucleotide and predicted amino acid sequence of zebrafish gdf7. The ORF contains two potential proteolytic processing motifs (R-X-X-R); site 1 (boxed and shaded) and site 2 (boxed). Three $\mathrm{N}$-linked glycosylation sites are located within the prodomain (underlined and in italics) and the mature domain contains seven conserved cysteine residues (circled). The sequence of the putative $3^{\prime}$ UTR contains two potential polyadenylation signals (thick underlined). (Arrowhead) Boundary between sequence obtained by 5' RACE and sequence obtained from genomic DNA. The GenBank accession nos. for zebrafish gdf7 are AF113022 and 
signal sequence. Repeated attempts of 5' RACE were not successful in extending the coding sequence further. Therefore, the complete coding sequence for $g d f 7$ remains to be isolated. Three consensus sites for Nlinked glycosylation were identified in the prodomain followed by two potential processing sites that conform to the R-X-X-R motif. Proteolytic cleavage at these sites would predict mature GDF7 peptides of 130, 128, or 127 amino acids (site 1; boxed and shaded in Fig. 1) and 102 amino acids (site 2; boxed in Fig. 1), respectively. Two cleavage sites in similar positions are found within human GDF7 and would give rise to mature peptides of 129 or 104 amino acids (Wolfman et al. 1997). Similarly, murine GDF7 has two cleavage sites, although these are more distantly separated by a glycine rich insert (Storm et al. 1994). Both of the human forms have been expressed in E. coli and are reported to be biologically active (Wolfman et al. 1997). The mature domain encoded by $g d f 7$, comprised of 113 conserved carboxy-terminal amino acids, was compared with the mature domains from mouse GDF5, GDF6, and GDF7 and found to share $81 \%, 88 \%$, and $82 \%$ amino acid identities, respectively. Two other genes belonging to the zebrafish $G d f 5,6,7$ subgroup, radar and dynamo, also encode mature domains that are most similar to GDF6 with $92 \%$ and $88 \%$ amino acid identity, respectively (Rissi et al. 1995; Bruneau et al. 1997). On the basis of these sequence comparisons, the orthologous identities of gdf7, radar, dynamo, and contact are not clear. Therefore, a neighbor-joining bootstrap tree was constructed to more accurately determine the evolutionary relationships between the zebrafish and mammalian genes (Fig. 2). On the basis of this analysis, contact groups with human GDF5 and murine $G d f 5$ with high bootstrap support that is consistent with the suggestion that contact is the zebrafish ortholog of Gdf5 (Bruneau et al. 1997). The radar and dynamo genes are most closely related to each other and are equally related to mammalian Gdf6 orthologs. The orthologous identity of $g d f 7$ cannot be unambiguously determined from the gene tree.

\section{Genetic Mapping and Analysis of Shared Syntenies}

To map $g d f 7$, we identified a restriction fragment length polymorphism (RFLP) in the putative $3^{\prime}$ untranslated region (UTR) and used this to localize the gene to linkage group (LG) 17 (Fig. 3). LG17 shares syntenies with three mouse chromosomes
(Mmu); Mmu14 (bmp4/Bmp4 and otx2/Otx2), Mmu2 (snap25.2/Snap25 and axial/Hnf3ß), and Mmu12 (gsc/ Gsc, pax9/Pax9, nk2.2/Nkx2-1, fkd7/Hnf3 $\alpha$, and sox11a/ Sox11; Fig. 4A). Mmu12 also contains Gdf7. LG17 shares syntenies with three human chromosome (Hsa) arms; Hsa2p (otx1/OTX1 and sox11a/SOX11; S.T. De Martino, T. Jowett, Y. Yan, J. Postlethwait, A. Ashworth and C.A. Austin, unpubl.), Hsa20p (snap25.2/SNAP25 and axial/HNF3 $\beta$ ) and Hsa14q (bmp4/BMP4, gsc/GSC, pax9/PAX9, nk2.2/NKX2A, otx2/OTX2, and fkd7/ $H N F 3 \alpha$; Postlethwait et al. 1998; Fig. 4A,B). To map human GDF7, primers were designed to the human sequence (A.J. Celeste, unpubl.) and scored on the Stanford Human Radiation Hybrid G3 mapping panel. GDF7 mapped to the short arm of Hsa2 near marker SHGC-33991 (lod score 12.13, cR_10,000, 5.02 cR). SHGC-33991 is in the interval from 40.7 to $48.5 \mathrm{~cm}$ that places it at the cytological postion 2p22-p21. Therefore, the three zebrafish/human gene pairs $g d f 7 /$

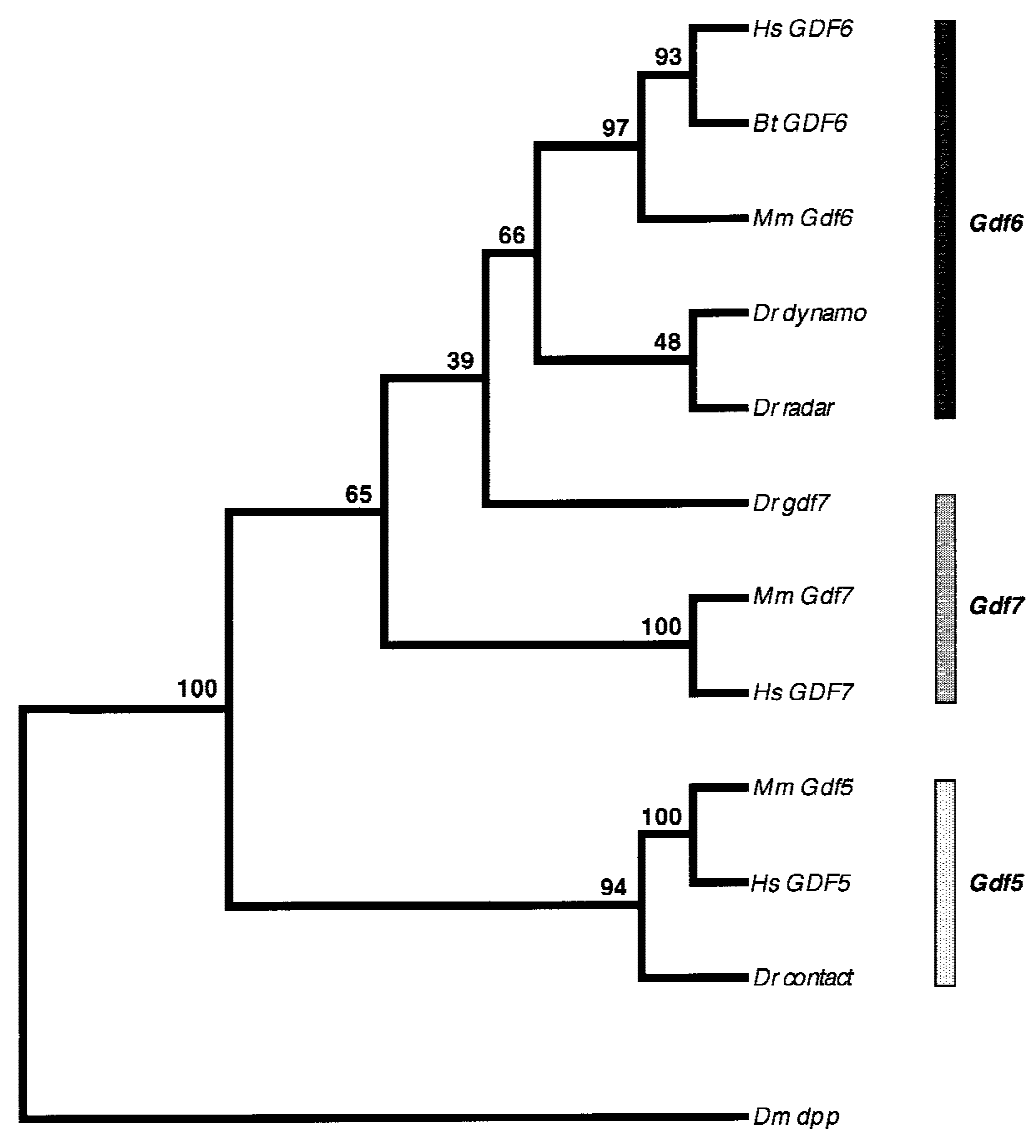

Figure 2 Phylogenetic relationships among members of the zebrafish and mammalian Gdf5, 6, 7 subgroup of genes. A neighbor-joining bootstrap tree was generated from mature domain sequences and arbitrarily outgrouped to $d p p$. Numbers above each branch point indicate percent bootstrap values (1000 replications). On the basis of this analysis and the genetic mapping (Fig. 3), the genes can be classified into three orthology groups: Gdf5, Gdf6, and Gdf7 as indicated by the three shaded bars. (Bt) Bos taurus; (Dr) Danio rerio; (Hs) Homo sapiens; (Mm) Mus musculus; (Dm) Drosophila melanogaster. 
A $\quad$ gdf7

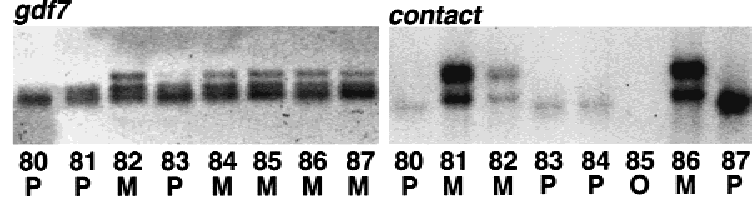

B LG17

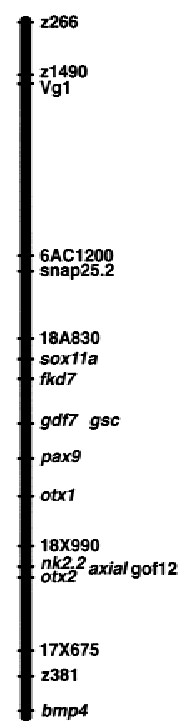

Figure 3 Genetic mapping of gdf7 and contact. (A) A RFLP was identified for $g d f 7$ by the restriction endonuclease Taq 1 . SSCP analysis was used to detect a polymorphism in the contact gene. Below each panel are the genotypes ( $\mathrm{M}$, maternal allele; $\mathrm{P}$, paternal allele; $\mathrm{O}$, missing data) of eight selected $\mathrm{F}_{2}$ progeny (numbers 80-87) from the mapping panel. (B) Genetic maps of LG17 and LG6 showing the location of gdf7 and contact. Cloned genes are written in italics, microsatellite markers begin with $z$ or gof and RAPD markers begin with a number (Postlethwait et al. 1994; Knapik et al. 1996, 1998; Postlethwait et al. 1998).

GDF7 (2p22-p21), sox11a/SOX11 (2p25), and otx1/ OTX1 (2p13) form a conserved synteny comprising part of Hsa2p and the central portion of LG17. On the basis of the shared syntenies in the mammalian and zebrafish genomes, it is likely that the zebrafish $g d f 7$ gene is the ortholog of GDF7/Gdf7.

A polymorphism in contact was identifed by singlestrand conformation polymorphism (SSCP) analysis and used to localize this gene to LG6 (Fig. 3), which also contains the genes brn1.2, ehh, and nic1 (Fig. 4B). LG6 and LG9 appear to be duplicate chromosomes (Amores et al. 1998; Postlethwait et al. 1998) and contain the genes brn1.1 and brn1.2, respectively, which are equally related to mouse Brn1 (Sampath and Stuart 1996). This suggests that the duplication event that generated LG6 and LG9 arose after the divergence of ray-finned (modern bony fish ancestor) and lobefinned (tetrapod ancestor) fish $\sim 420$ million years ago (Ahlberg and Milner 1994). LG9 contains a number of other loci that have mammalian orthologs on Hsa2q, Mmu1, and Mmu2 (Fig. 4B). LG6 contains nic1, which encodes the nicotinic acetylcholine receptor- $\alpha$ (Sepich et al. 1998), and is the zebrafish ortholog of human CHRNA1 on Hsa2q and mouse Acra on Mmu2. Mmu2 also contains $G d f 5$, which is likely to be orthologous to contact, on the basis of the phylogenetic analysis (Fig. 2 ). Taken together, the mapping data suggests that an ancient chromosome may have contained the progenitors of contact/GDF5/Gdf5, bmp2b/BMP2/Bmp2, snap25.1/SNAP25/Snap25, PAX1/Pax1, HNF3 $\beta / H n f 3 \beta$, brn1.1/brn1.2/Brn1, hoxd4/HOXD4/Hoxd4, evx2/EVX2/ Evx2, eng1/EN1/En1, dlx2/DLX2/Dlx2, des/DES/Des, ehh/ hha/IHH/Ihh, actr2/Acvr2a, actbb/INHBB/Inhbb, dermo1/ DERMO1, and nic1/CHRNA1/Acra. In the human lineage, this was broken into two segments found on Hsa20 and Hsa2q. In the mouse chromosome, after some inversions that altered the gene order with respect to humans, it was also broken into two chromosomes, but in a different place than in the human lin-

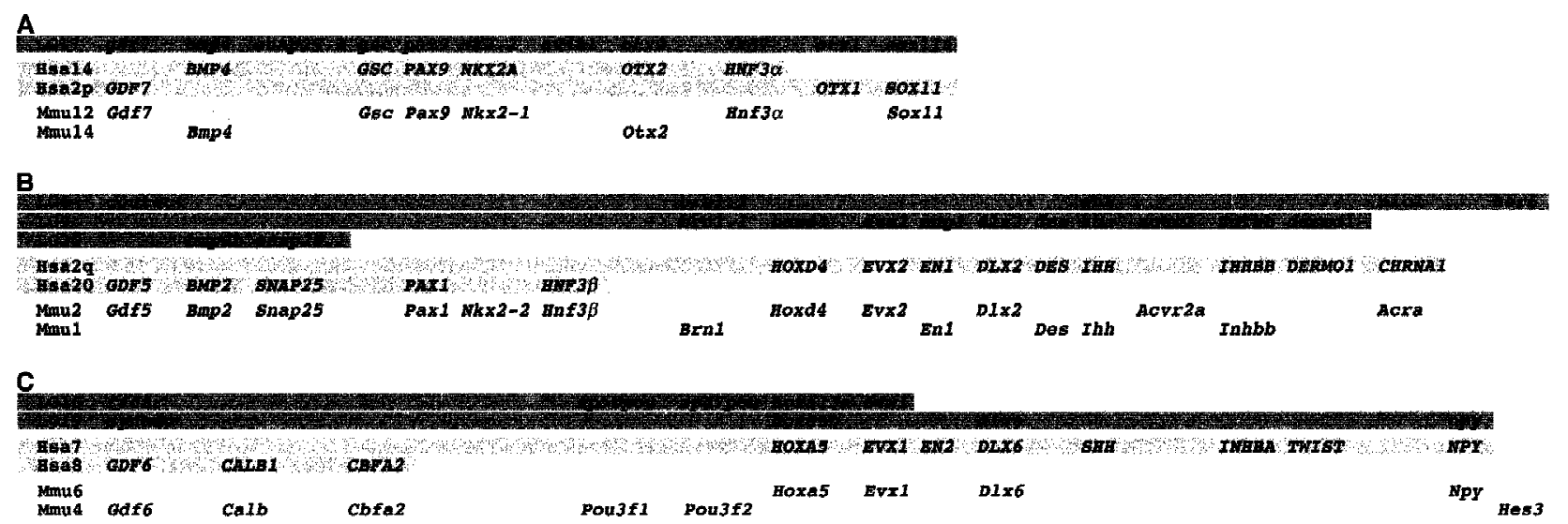

Figure 4 Comparative genetic mapping of the $G d f 5,6,7$ subgroup of genes in zebrafish and mammals. $(A)$ Gdf7-bearing chromosome group. (B) Gdf5-bearing chromosome group. (C) Gdf6-bearing chromosome group. Each row shows the contents of different zebrafish (LG), human ( $\mathrm{Hsa})$, or mouse (Mmu) chromosomes. For ease of comparison, the genes have been arbitrarily ordered so that members of gene families are displayed in columns. Within a chromosome group the genes in columns are apparent orthologs, or in the case of some zebrafish chromosomes, paralogs resulting from a genome duplication not shared with tetrapods. 
eage, thereby giving Mmu1 and Mmu2. In the zebrafish lineage, the ancestral chromosome or at least a segment of it, was duplicated, eventually giving rise to portions of LG6 and LG9. The segment of LG20 that contains bmp2b and snap25.1 probably became separated from either LG6 or LG9 by translocation.

The genes radar and dynamo have been localized to LG16 and LG19, respectively (Postlethwait et al. 1998). The phylogenic analysis (Fig. 2) suggests that radar and dynamo are most closely related to each other and are equally related to mammalian GDF6/Gdf6. Therefore, radar and dynamo may be duplicate genes that are orthologous to $G D F 6 / G d f 6$. The mapping data further supports this conclusion. LG16 contains radar, zp50pou, zp47pou, hoxa13a, and evx1 (Amores et al. 1998; Postlethwait et al. 1998; Fig. 4C). The murine orthologs of zp50pou and zp47pou are found on Mmu4 (Avraham et al. 1993; Hauptmann and Gerster 1996; Spaniol et al. 1996), which also contains Gdf6. Therefore, these conserved syntenies are consistent with $\mathrm{ra}$ dar being orthologous to murine $G d f 6$. LG19 contains dynamo, hoxa5b, dlx6, and npy (Fig. 4C; Amores et al. 1998; Postlethwait et al. 1998). Gene phylogenies show that the hoxaa and hoxab loci in zebrafish are paralogs that appear to have been derived from a chromosomal duplication in the teleost lineage (Amores et al. 1998). The linkage of radar and dynamo to paralogous hox clusters is consistent with the suggestion that these zebrafish Gdf5, 6, 7 subgroup members are duplicate orthologs of murine Gdf6. To map human GDF6, primers were designed to the human sequence (A.J. Celeste, unpubl.) and scored on the Genebridge4 radiation hybrid panel. GDF6 mapped to Hsa8 near marker WI9077 (lod score >15, cR_3000, $5.23 \mathrm{cR}$ ), which corresponds to the gene ATP6D (UniGene Hs.86905). WI9077 maps in the interval from D8S270 (102.1 cM) to D8S257 $(110.3 \mathrm{~cm})$ on the human gene map that corresponds to the cytogenetic position $8 \mathrm{q} 21.3$. This region of Hsa8 shares syntenies with Mmu4 including the genes CBFA2/Cbfa2 and CALB1/Calb, consistent with the chromosomal location of $G d f 6$ in the mouse Fig. 4C). The human orthologs of zp50pou/Pou3f1 and zp47pou/Pou3f2 are found on 1p34.1 and 6q16, respectively (Sumiyama et al. 1998; Atanasoski et al. 1995), suggesting that these genes became dispersed from GDF6, $C B F A 2$, and CALB1 by independent rearrangements in the human lineage.

In comparing Figure $4, \mathrm{~A}$ and $\mathrm{B}$, notice that the genes GDF5/Gdf5, BMP2/Bmp2, PAX1/Pax1, and Nkx2-2 on Hsa20 and Mmu2 are paralogous to GDF7/ Gdf7, BMP4/Bmp4, PAX9/Pax9, and NKX2A/Nkx2-1 on Hsa2p or Hsa14 and Mmu12 or Mmu14. This suggests that an ancient duplication event that occurred prior to the divergence of the ray-finned and lobe-finned fishes produced two duplicate chromosome segments. Independent rearrangements then occurred in the mu- rine and human lineages resulting in these segments becoming portions of Hsa20, Hsa14, Hsa2p, and Mmu2, Mmu12, and Mmu14.

\section{Expression of gdf7}

The expression of $g d f 7$ during embryonic development and in adult tissues was examined by RT-PCR by use of $g d f 7$-specific primers (Fig. 5). Developmental stages from $5 \mathrm{hr}$ postfertilization (hpf) to 5 days postfertilization and the organs brain, heart, liver, kidney, gut, muscle, testis, and ovary were examined. The PCR products were analyzed by Southern hybridization with an internal radiolabeled oligonucleotide specific to $g d f 7$. Expression of $g d f 7$ was weakly detected at $5 \mathrm{hpf}$ and then at every stage examined through to $120 \mathrm{hpf}$. Expression of $g d f 7$ was detected in all of the adult tissues examined but was most readily amplified from cDNA isolated from the brain, liver, kidney, muscle, testis, and ovary. No RT-PCR products were detected in control samples that were not treated with reverse transcriptase (data not shown). As a positive control, the same cDNAs were amplified with $\beta$-actin-specific primers and also hybridized to an internal oligonucleotide.

The expression of $g d f 7$ at $48 \mathrm{hpf}$ was examined by whole mount in situ hybridization (Fig. 6). Transcripts for $g d f 7$ were detected in dorsal and ventral regions of the head, the pronephric ducts, and the dorsal aorta in the trunk of the embryo. In the head, the dorsal population of cells formed two bilateral stripes that appeared coincident with the location of the paired trabeculae of the developing neurocranium (Schilling et al. 1996; Schilling and Kimmel 1997). The more ventrally located cells that express $g d f 7$ are likely to represent precartilaginous condensations of the palatoquadrates, ceratohyals, and Meckel's cartilage, which com-

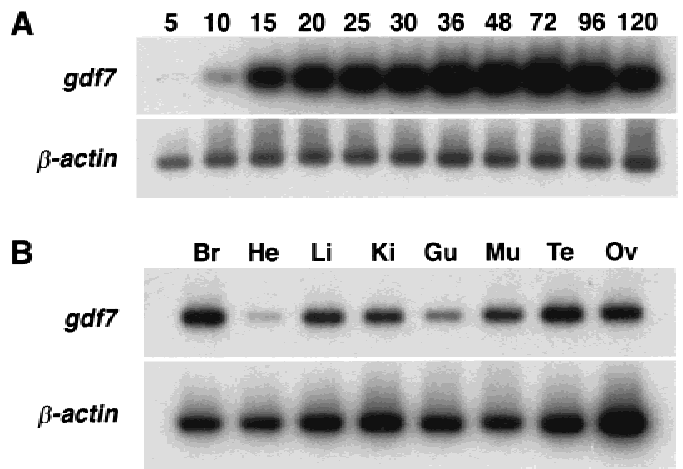

Figure 5 Embryonic and adult expression of $g d f 7$. Total RNA was isolated from zebrafish embryos at selected stages from $5 \mathrm{hpf}$ to $120 \mathrm{hpf}(A)$ and from adult tissues $(B)$. The expression of $g d f 7$ was examined by RT-PCR and the amplified products were detected following hybridization of an internal radiolabeled gdf7specific oligonucleotide. The expression of $\beta$-actin was examined as a positive control for cDNA synthesis. (Br) brain; (He) heart; (Li) liver; (Ki) kidney; (Gu) gut; (Mu) muscle; (Te) testis; (Ov) ovary. 


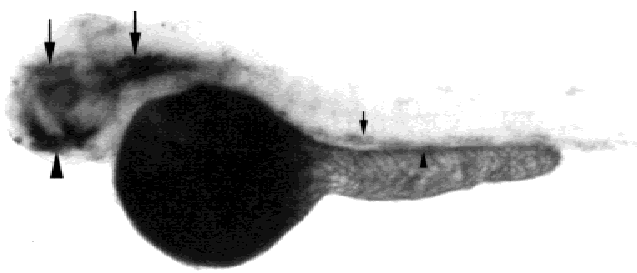

Figure 6 Expression of $g d f 7$ at $48 \mathrm{hpf}$ by whole mount in situ hybridization. A lateral view of a $48 \mathrm{hpf}$ embryo is shown with anterior toward the left and dorsal toward the top of the page. Transcripts for $g d f 7$ can be detected in two bilateral stripes of cells in regions of the developing neurocranium (large arrows) and in precartilage condensations of the developing jaw (large arrowhead). Expression of gdf7 is also observed in the dorsal aorta (small arrow) and in the pronephric ducts (small arrowhead). The staining in the yolk is nonspecific.

prise the jaw and supporting elements (Schilling et al. 1996; Schilling and Kimmel 1997). A more detailed examination of $g d f 7$ expression during craniofacial development and how it compares with other members of the zebrafish $G d f 5,6,7$ subgroup is currently under way.

\section{DISCUSSION}

We have identified $g d f 7$, a fourth gene member of the zebrafish Gdf5, 6, 7 subgroup. The predicted amino acid sequence encoded by $g d f 7$ contains potential processing sites and seven conserved cysteine residues characteristic of members of the TGF- $\beta$ superfamily. An analysis of gene expression by RT-PCR showed that $g d f 7$ is an active gene that is expressed during embryonic development and in adult tissues. Expression of gdf7 in 48 hpf embryos was examined by whole mount in situ hybridization and transcripts were predominantly detected in regions of the head undergoing cartilage development.

The finding that the zebrafish genome contains four genes that belong to the Gdf5, 6, 7 subgroup prompted us to explore the evolutionary origin of these genes by comparative genetic mapping. Such an approach provides an alternative basis on which to infer evolutionary relationships among distantly related phyla. The $g d f 7$ gene mapped to LG17, which shares syntenic relationships with genes found on Mmu12, including Gdf7. Similarly, LG17 shares syntenies with Hsa2p that includes the GDF7 gene. Thus, it is likely that $g d f 7$ is the zebrafish ortholog of GDF7/Gdf7. Additional support for this orthologous assignment is the presence of two potential cleavage sites in the prodomain of zebrafish GDF7 that are also found conserved in human and mouse GDF7 sequences but are not found within human or mouse GDF5 or GDF6 (Storm et al. 1994; Wolfman et al. 1997). Furthermore, the partial prodomain sequence of zebrafish GDF7 shows the greatest similarity to the prodomain of human GDF7 (data not shown). The contact gene is most closely related to mammalian GDF5/Gdf5 genes and its chromosomal localization to LG6 is consistent with it being the zebrafish ortholog of GDF5/Gdf5. The mapping data suggests LG6 and LG9 are duplicate chromosomes and a translocation has separated the genes $b m p 2 b$ and snap25.1 from either LG6 or LG9. Another ortholog of $b m p 2$ exists in zebrafish, termed bmp2a (Martínez-Barberá et al. 1997) and maps to LG17 (J.H. Postlethwait, unpubl.), which also contains snap25.2. This data suggests that parts of LG17 and LG20 are duplicate chromosome segments and raises the possibility that another contact gene may also exist. If so, it would likely map to either LG9, LG17, or LG20.

It has been suggested that dynamo may represent a new member of the Gdf5, 6, 7 subgroup that has yet to be identified in higher vertebrates (Bruneau and Rosa 1997). However, our data suggests an alternative hypothesis. The radar and dynamo genes appear as sister groups on the gene tree and are closely related to mammalian GDF6/Gdf6 orthologs. Genetic mapping localized radar and dynamo to LG16 and LG19, respectively, and previous mapping data suggests that these are duplicate chromosomes as they each contain one copy of duplicate hoxa complexes (Amores et al. 1998). LG16 shares syntenic relationships with genes found on Mmu4, including Gdf6. Therefore, it is likely that $d y$ namo and radar both represent orthologs of murine Gdf6 that have arisen from a chromosomal duplication that has occurred in the zebrafish lineage. Members of the $G d f 5,6,7$ subgroup share a similar gene structure comprised of two coding exons (A.J Celeste, unpubl.). The location of the intron separating the two coding exons is specific for each subgroup member. The position of the intron separating the two coding exons in radar and dynamo is conserved, consistent with the suggestion that these are duplicated genes (A.J. Davidson, unpubl.). Thus, we would predict that an additional Gdf5, 6, 7 member corresponding to the ortholog of dynamo will not be found in mammals. Extensive genomic library screening has yet to reveal a dynamo ortholog in humans (A.J. Celeste, unpubl.).

It is believed that gene duplication by polyploidization has been an important part of vertebrate evolution and a major contributor to multigene families (Ohno 1970; Holland et al. 1994; Sidow 1996; Postlethwait et al. 1998). At least two genome duplications have occurred during the evolution of vertebrates, both happening prior to the divergence of rayfinned and lobe-finned fish (Holland et al. 1994; Amores et al. 1998; Postlethwait et al. 1998). A comparison of shared syntenies in this study suggests that the loci $g d f 7 / G D F 7 / G d f 7$ are paralogs of contact/GDF5/ Gdf5 and probably arose from one of these rounds of vertebrate polyploidization. However, it is apparent from the mapping data that additional chromosome duplications have since occurred during zebrafish evo- 
lution, such as those that generated the LG6/LG9 and LG16/LG19 pairs of chromosome segments. Extra members of other developmental multigene families in zebrafish have also been reported (Akimenko et al. 1995; Ekker et al. 1995; Stock et al. 1996; Zardoya et al. 1996) and in some cases these extra genes are found in duplicated chromosome segments (Postlethwait et al. 1998). A comprehensive screen for zebrafish hox genes and the subsequent mapping of these genes has revealed that an additional genome duplication event not shared with tetrapods is likely to have occurred prior to teleost radiation (Amores et al. 1998). Such a tetraploidization event would explain the greater number of paralogous gene copies found in zebrafish and provides a mechanism for the origin of the gene duplicates radar and dynamo.

By determining the orthologous assignments of gdf7, radar, dynamo, and contact it will now be possible to interpret more meaningfully a comparison of expression patterns between zebrafish and mammals that should yield additional insights into the function of these genes during development. Furthermore, the mapping of these genes will aid in the identification of candidates for some of the mutations recently generated in zebrafish, particularly those involved in cartilage and bone formation. Finally, in line with agreed on nomenclature (Westerfield 1994) and to avoid further confusion resulting from orthologs having multiple names, we propose that radar, dynamo, and contact be renamed $g d f 6 a, g d f 6 b$, and $g d f 5$, respectively.

\section{METHODS}

\section{Isolation of Zebrafish gdf7}

A 291-bp PCR fragment encoding most of the mature domain of murine GDF7 (Wolfman et al. 1997) was radioactively labeled by random priming (Boehringer Mannheim Australia, NSW) and used as a probe to screen $\sim 1 \times 10^{6}$ plaques of a zebrafish genomic DNA library (Stratagene, La Jolla, CA). Hybridization was carried out according to standard procedures (Sambrook et al. 1989 ) at $65^{\circ} \mathrm{C}$ in $5 \times$ SSC, $5 \times$ Denhardt's solution, $0.5 \%$ SDS and $100 \mu \mathrm{g} / \mathrm{ml}$ denatured salmon sperm DNA. The membranes (Hybond-N+; Amersham, Buckinghamshire, England) were washed to a final stringency of $2 \times$ SSC, $0.1 \%$ SDS at $65^{\circ} \mathrm{C}$. Hybridizing plaques were picked and purified by two additional rounds of screening. Four distinct positive phage clones corresponding to gdf7, radar, dynamo, and contact were identified and the sequences that hybridized to the probe were subcloned into pBluescript SK- (Stratagene, La Jolla, CA) and sequenced. Additional sequence of $g d f 7$ was identified by use of total RNA extracted from adult testis tissue and the 5' RACE system (GIBCO-BRL, Gaithersburg, MD) according to the manufacturer's instructions.

\section{DNA Sequencing}

DNA sequencing of both strands was done with the PRISM Ready Reaction kits with AmpliTaq FS DNA Polymerase (Perkin Elmer, Foster City, CA), following the manufacturer's recommendations for template and primer concentrations and cycling conditions. M13 forward and reverse primers or synthetic oligonucleotide primers were used. Reactions were run in the 9600 GeneAmp PCR system (Perkin Elmer, Foster City, CA) or the PTC200 Peltier Thermal Cycler (MJ Research, Watertown, MA). Sequencing reactions were purified by 96-well gel filtration blocks (AGCT Technologies, Gaithersburg, MD) and then resolved on an acrylamide gel with the Perkin Elmer 373 DNA analysis system. Sequence assembly and editing was performed on Apple Macintosh computers with the Sequencher program (Gene Codes, Ann Arbor, MI).

\section{Phylogenetic Analysis}

Amino acid sequences of the following proteins (GenBank accession nos. or the literature source are given in parentheses) were aligned by use of the software package MacVector (Oxford Molecular Group, Oxford, England): Radar (Rissi et al. 1995); zebrafish GDF7 (present study); Dynamo (X99769); Contact (Y12005); bovine GDF6 (also known as CDMP2; P55106); Decapentaplegic (U63857); murine GDF5, 6, 7 (U08337-U08839); human GDF5 (also known as CDMP1; P43026); and human GDF6 and GDF7 (Wolfman et al. 1997; additional unpublished sequence was provided by A.J Celeste). The alignment started 11 amino acids from the first conserved cysteine residue through to the last carboxyterminal amino acid residue (113 characters). The phylogenetic analysis was performed with the Phylogenetic Inference Package, PHYLIP 3.5 (Felsenstein 1993). SEQBOOT was used to resample the alignment (1000 bootstrap replications). Distance matrices were generated by PROTDIST, on the basis of the Dayhoff PAM model of amino acid substitution. Neighbor-joining trees were calculated by NEIGHBOR and a consensus tree was derived by CONSENSE. Decapentaplegic (DPP) was used as an arbitrary outgroup for the tree.

\section{Genetic Mapping}

The gdf7 gene was mapped by the identification of a RFLP. Primers specific to the putative 3' UTR of $g d f 7$ were used to amplify a 221-bp fragment by use of genomic DNA from C32 or SJD parental strains as a template (a gift from L.I. Zon, Children's Hospital, Boston, MA). The PCR reaction mixture contained $200 \mathrm{ng}$ of genomic DNA, $1 \times$ PCR buffer (Perkin Elmer, Norwalk, CT), $1.5 \mathrm{~mm} \mathrm{MgCl}_{2}, 0.2 \mathrm{~mm}$ each dNTP, 2.5 units of AmpliTaq DNA Polymerase (Perkin Elmer, Norwalk, CT), and $1 \mu \mathrm{M}$ of each primer (upper primer $5^{\prime}$ ATGACACCACATTTGGCTTGGG-3'; lower primer 5' CACACCCTCTCAGTCAATGTAG-3'). Amplification was for 40 cycles of $1 \mathrm{~min}$ at $94^{\circ} \mathrm{C}, 1 \mathrm{~min}$ at $55^{\circ} \mathrm{C}$, and $1 \mathrm{~min}$ at $72^{\circ} \mathrm{C}$. The PCR products were subcloned into pCR2.1 (Invitrogen, San Diego, CA) and sequenced. A single base pair polymorphism was found that generated an additional Taq1 restriction enzyme site within the SJD amplified fragment.

For SSCP analysis, genomic DNA from C32 and SJD parental strains was amplified with primers specific to the $3^{\prime}$ UTR of contact (upper primer 5'-GTACGAGGACATGGTGGTGGAGAG-3'; lower primer 5'-TCGGAATGGAACTGAGTGAGAATG-3'). One of the primers was end-labeled by use of $\left[\gamma{ }^{32} \mathrm{P}\right]$ ATP $(6000 \mathrm{Ci} / \mathrm{mmole}$; Amersham) and T4 polynucleotide kinase (GIBCO-BRL, Gaithersburg, MD). The PCR reaction mixture contained $250 \mathrm{ng}$ of template DNA, $10 \mathrm{~mm}$ Tris$\mathrm{Cl}$ ( $\mathrm{pH} 8.3$ ), $50 \mathrm{~mm} \mathrm{KCl}, 2 \mathrm{~mm} \mathrm{MgCl}_{2}, 0.2 \mathrm{~mm}$ of each dNTP, $1 \mu \mathrm{M}$ of each primer and 1.0 units of Taq DNA polymerase in a final volume of $12 \mu \mathrm{l}$. The reaction mixture was initially denatured at $94^{\circ} \mathrm{C}$ for $5 \mathrm{~min}$. Amplification was for 30 cycles 
of $1 \mathrm{~min}$ at $94^{\circ} \mathrm{C}, 2 \mathrm{~min}$ at $55^{\circ} \mathrm{C}$ and $3 \mathrm{~min}$ at $72^{\circ} \mathrm{C}$. There was a final extension of $7 \mathrm{~min}$ at $72^{\circ} \mathrm{C}$. One-sixth of the sample was mixed with $8 \mu \mathrm{l}$ of Stop solution (100\% formamide, $0.25 \%$ Bromophenol Blue, 0.25\% Xylene Cyanol FF), denatured for $5 \mathrm{~min}$ at $94^{\circ} \mathrm{C}$ and then chilled on ice before being loaded onto a $5 \%$ nondenaturing polyacrylamide gel containing $0.5 \times$ TBE buffer. The gel was electrophoresed at $4^{\circ} \mathrm{C}$ in $0.5 \times$ TBE buffer at $40 \mathrm{Watts}$. The gel was transferred and dried onto Whatman $3 \mathrm{MM}$ paper before being exposed to X-ray film at $-70^{\circ} \mathrm{C}$ overnight.

The RFLP and SSCP identified for $g d f 7$ and contact were genotyped on DNA from $96 \mathrm{~F}_{2}$ progeny from the linkage map cross that has been genotyped previously for $>650$ PCR-based markers (Postlethwait et al. 1994; Johnson et al. 1996; Knapik et al. 1996, 1998; Postlethwait et al. 1998). The strain distribution patterns were analyzed by the program LINKER (Postlethwait et al. 1994) and maps were constructed with MAPMAKER (Lander 1987). The locations of mammalian gene loci were taken from the Mouse Genome Database (http://www.informatics.jax.org/), the Online Mendelian Inheritance of Man (http://gdbwww.gdb.org/omim/docs/ omimtop.html), the Genome Database (http:// gdbwww.gdb.org/gdb), and The Human Transcript Map (http://www.ncbi.nlm.nih.gov/SCIENCE96/).

To map human GDF7, the primers 5'-GGATAGCCCGGGCGAAGACG-3' and 5'-GCGGGGCCTCCTAACAGCAAATG-3' were designed and found to amplify human, but not hamster, DNA from the Stanford Human Radiation Hybrid G3 Panel obtained from Research Genetics, Inc. DNAs from panel members were amplified by PCR and displayed on $2 \%$ agarose gels. Gels were scored and the results submitted to the Stanford Human Genome Center for mapping (http:// www-shgc.stanford.edu/Mapping/rh/). To map human GDF6, two independent primer pairs (GDF6a: upper primer 5'ACAAGCAGTACGAGGACATGG-3', lower primer 5' ATCCAGGCTGTTCCCTCAC-3'; GDF6b: upper primer 5'AGAGAGGCGGAGGAGGAAG-3', lower primer 5' GGGCTGGCAGGTAGAAGTTAC-3') were tested with the Genebridge4 radiation hybrid panel obtained from Research Genetics, Inc. Samples that gave concordant results with these primers were scored and the data analyzed by use of the Whitehead Institute radiation hybrid mapping web interface (http://carbon.wi.mit.edu:8000/cgi-bin/contig/rhmapper.pl).

\section{RT-PCR and Whole Mount in Situ Hybridization}

Total cellular RNA was isolated from embryos at 5, 10, 15, 20, $25,30,36,48,72,96$, and $120 \mathrm{hpf}$ and from adult tissues with TRIZOL (GIBCO-BRL, Gaithersburg, MD) according to the manufacturer's instructions. First strand cDNA was synthesized from each stage with Superscript II reverse transcriptase (GIBCO-BRL, Gaithersburg, MD) in a reaction volume of 20 $\mu \mathrm{l}$ according to the manufacturer's instructions. To control for genomic DNA contamination, the samples were also incubated in the absence of reverse transcriptase. PCR was performed in a reaction volume of $50 \mu \mathrm{l}$ containing $3 \mu \mathrm{l}$ of cDNA, $1 \times$ PCR buffer (Perkin Elmer), $1.5 \mathrm{~mm} \mathrm{MgCl}_{2}, 0.2 \mathrm{~mm}$ of each dNTP, 2.5 units of AmpliTaq DNA Polymerase (Perkin Elmer, Norwalk, CT) and $1 \mu \mathrm{M}$ of each $g d f 7$-specific primer (upper primer 5'-TGGAAGACGGAGGACACG-3'; lower primer 5'CTACCTGCACCCACAACT-3') or $\beta$-actin-specific primer (upper primer 5'-GTCGTCGACAACGGCTCCGGCATGTG-3'; lower primer $5^{\prime}$-CATTGTAGAAGGTGTGGTGCCAGAT-3'). Amplification was for 28 cycles of $1 \mathrm{~min}$ at $94^{\circ} \mathrm{C}, 1 \mathrm{~min}$ at $57^{\circ} \mathrm{C}$ and $1.5 \mathrm{~min}$ at $72^{\circ} \mathrm{C}$ generating a 328-bp gdf7 product or a 253-bp $\beta$-actin product. The PCR products were resolved on a $1.5 \%$ agarose gel, blotted to nylon membranes (Hybond$\mathrm{N}+$; Amersham), and hybridized to an end-labeled internal oligonucleotide ( $g d f 7$-specific $5^{\prime}$-CAGAGTCCACCCCTCCC-3'; $\beta$-actin-specific 5'-GGACAGAAAGACAGCTACGT-3') according to established procedures (Sambrook et al. 1989).

Whole-mount in situ hybridization was performed as described (Schulte-Merker et al. 1992). The gdf7 riboprobe was labeled with digoxigenin and detection of the antidigoxigenin antibody-alkaline phosphatase conjugate was done with 4-nitroblue tetrazolium chloride (NBT) and 5-bromo-4chloro-3-indolyl-phosphate (BCIP). After whole-mount in situ hybridization, the embryos were refixed in $4 \%$ paraformaldehyde, transferred into $80 \%$ glycerol, and photographed.

\section{ACKNOWLEDGMENTS}

We thank the following people: Maria Vitas and Scott Mead for technical assistance; Kevin Bean for DNA sequencing; Dr Hazel Sive, Dr Leonard Zon, Steve Clark, Rod Hewick, Vicki Rosen, Jenn Dube, and Beth Murray for advice and assistance. This work was supported by a University of Auckland Doctoral Scholarship (A.J.D.), an Auckland Medical Research Foundation Senior Scholarship (A.J.D) and grants R01RR10715 (J.H.P) and PHS P01HD22486 (J.H.P).

The publication costs of this article were defrayed in part by payment of page charges. This article must therefore be hereby marked "advertisement" in accordance with 18 USC section 1734 solely to indicate this fact.

\section{REFERENCES}

Ahlberg, P.E. and A.R. Milner. 1994. The origin and early diversification of tetrapods. Nature 368: 507-514.

Akimenko, M.A., S.L. Johnson, M. Westerfield, and M. Ekker. 1995. Differential induction of four $m s x$ homeobox genes during fin development and regeneration in zebrafish. Development 121: $347-357$.

Amores, A., A. Force, Y.-Y. Yan, L. Joly, C. Amemiya, A. Fritz, R.K. Ho, J. Langeland, V. Prince, Y.-L. Wang, M. Westerfield, M. Ekker, and J.H. Postlethwait. 1998. Zebrafish hox clusters and vertebrate genome evolution. Science 282: 1711-1714.

Atanasoski, S., S.S. Toldo, U. Malipiero, E. Schreiber, R. Fries, and A. Fontana. 1995. Isolation of the human genomic brain-2/N-Oct 3 gene (POUF3) and assignment to chromosome 6q16. Genomics 26: $272-280$.

Avraham, K.B., B.C. Cho, D. Gilbert, H. Fujii, K. Okamoto, T. Shimazaki, T. Ito, H. Shoji, Y. Wakamatsu, H. Kondoh et al. 1993. Murine chromosomal location of four class III POU transcription factors. Genomics 18: 131-133.

Bruneau, S. and F.M. Rosa. 1997. Dynamo, a new zebrafish DVR member of the TGF- $\beta$ superfamily is expressed in the posterior neural tube and is up-regulated by sonic hedgehog. Mech. Dev. 61: 199-212.

Bruneau, S., P. Mourrain, and F.M. Rosa. 1997. Expression of contact, a new zebrafish DVR member, marks mesenchymal cell lineages in the developing pectoral fins and head and is regulated by retinoic acid. Mech. Dev. 65: 163-173.

Chang, S.C., B. Hoang, J.T. Thomas, S. Vukicevic, F.P. Luyten, N.J.P. Ryba, C.A. Kozak, A.H. Reddi, and M. Moos Jr. 1994. Cartilage-derived morphogenetic proteins. New members of the transforming growth factor- $\beta$ superfamily predominantly expressed in long bones during human embryonic development. J. Biol. Chem. 269: 28227-28234.

Daopin, S., K.A. Piez, Y. Ogawa, and D.R. Davies. 1992. Crystal structure of transforming growth factor- $\beta 2$ : An unusual fold for the superfamily. Science 257: 369-373. 
Dubois, C.M., M.H. Laprise, F. Blanchette, L.E. Gentry, and R. Leduc 1995. Processing of transforming growth factor $\beta 1$ precursor by human furin convertase. J. Biol. Chem. 270: 10618-10624.

Eigenbrot, C. and N. Gerber. 1997. X-ray structure of glial cell-derived neurotrophic factor at 1.9 A resolution and implications for receptor binding. Nat. Struct. Biol. 4: 435-438.

Ekker, S.C., A.R. Ungar, P. Greenstein, D.P. von Kessler, J.A. Porter, R.T. Moon, and P.A. Beachy. 1995. Patterning activities of vertebrate hedgehog proteins in the developing eye and brain. Curr. Biol. 5: 944-955.

Felsenstein, J. 1993. PHYLIP (Phylogeny Inference Package). Department of Genetics, University of Washington, Seattle, WA.

Griffith, D.L., P.C. Keck, T.K. Sampath, D.C. Rueger, and W.D. Carlson. 1996. Three-dimensional structure of recombinan human osteogenic protein 1: structural paradigm for the transforming growth factor $\beta$ superfamily. Proc. Natl. Acad. Sci. 93: $878-883$.

Hauptmann, G. and T. Gerster. 1996. Complex expression of the zp-50 pou gene in the embryonic zebrafish brain is altered by overexpression of sonic hedgehog. Development 122: 1769-1780.

Hogan, B.L. 1996. Bone morphogenetic proteins: Multifunctional regulators of vertebrate development. Genes \& Dev. 10: $1580-1594$.

Holland, P., J. Garcia-Fernàndez, N.A. Williams, and A. Sidow. 1994. Gene duplications and the origins of vertebrate development. Development (Suppl.) 125-133.

Inada, M., T. Katagiri, S. Akiyama, M. Namika, M. Komaki, A. Yamaguchi, K. Kamoi, V. Rosen, and T. Suda. 1996. Bone morphogenetic protein-12 and -13 inhibit terminal differentiation of myoblasts, but do not induce their differentiation into osteoblasts. Biochem. Biophys. Res. Comm. 222: 317-322.

Johnson, S.L., M.A. Gates, M. Johnson, W.S. Talbot, S. Horne, K. Baik, S. Rude, J.R. Wong, and J.H. Postlethwait. 1996. Centromere-linkage analysis and consolidation of the zebrafish genetic map. Genetics 142: 1277-1288.

Kingsley, D.M. 1994. The TGF- $\beta$ superfamily: New members, new receptors, and new genetic tests of function in different organisms. Genes \& Dev. 8: 133-146.

Knapik, E.W., A. Goodman, O.S. Atkinson, C.T. Roberts, M. Shiozawa et al. 1996. A reference cross DNA panel for zebrafish (Danio rerio) anchored with simple sequence length polymorphisms. Development 123: 451-460.

Knapik, E.W., A. Goodman, M. Ekker, M. Chevrette, J. Delgado et al. 1998. A microsatellite genetic linkage map for zebrafish (Danio rerio). Nat. Genet. 18: 338-343.

Krieglstein, K., C. Suter-Crazzolara, G. Hötten, J. Pohl, and K. Unsicker. 1995. Trophic and protective effects of growth/differentiation factor 5, a member of the transforming growth factor- $\beta$ superfamily, on midbrain dopaminergic neurons. J. Neurosci. Res. 42: 724-732.

Lander, E.S. 1987. MAPMAKER: An interactive computer package for constructing primary genetic linkage maps of experimental and natural populations. Genomics 1: 174-181.

Lee, K.J., Mendelsohn, M., and T.M Jessell. 1998. Neuronal patterning by BMPs: A requirement for GDF7 in the generation of a discrete class of commissural interneurons in the mouse spinal cord. Genes \& Dev. 12: 3394-3407.

Martínez-Barberá, J.P., H. Toresson, S. Da Rocha, and S. Krauss. 1997. Cloning and expression of three members of the zebrafish Bmp family: Bmp2a, Bmp2b, and Bmp4. Gene 198: 53-59.

Nachtigal, M.W. and H.A. Ingraham. 1996. Bioactivation of Müllerian inhibiting substance during gonadal development by a kex2/subtilisin-like endoprotease. Proc. Natl. Acad. Sci. 93: 7711-7716.

Ohno, S. 1970. Evolution by gene duplication. Springer-Verlag, Heidelberg, Germany.

Postlethwait, J.H., S.L. Johnson, C.N. Midson, W.S. Talbot, M. Gates, E.W. Ballinger, D. Africa, R. Andrews, T. Carl, J.S. Eisen et al. 1994. A genetic linkage map for the zebrafish. Science 264: 699-703.

Postlethwait, J.H., Y.-L. Yan, M.A. Gates, S. Horne, A. Amores, A.
Brownlie, A. Donovan, E.S. Egan, A. Force, Z. Gong et al. 1998. Vertebrate genome evolution and the zebrafish gene map. Nature Genet. 18: 345-349.

Rissi, M., J. Wittbrodt, E. Delot, M. Naegeli, and F.M. Rosa. 1995. Zebrafish Radar: A new member of the TGF- $\beta$ superfamily defines dorsal regions of the neural plate and the embryonic retina. Mech. Dev. 49: 223-234.

Sambrook, J., E.F. Fritsch, and T. Manniatis. 1989. Molecular cloning. A laboratory manual, 2nd edition. Cold Spring Harbor Laboratory Press, Cold Spring Harbor, NY.

Sampath, K. and G.W. Stuart. 1996. Developmental expression of class III and IV POU domain genes in the zebrafish. Biochem. Biophys. Res. Comm. 219: 565-571.

Schilling, T.F. and C.B. Kimmel. 1997. Musculoskeletal patterning in the pharyngeal segments of the zebrafish embryo. Development 124: $2945-2960$.

Schilling, T.F., T. Piotrowski, H. Grandel, M. Brand, C.-P. Heisenberg, Y.-J. Jiang, D. Beuchle, M. Hammerschmidt, D.A. Kane, M.M. Mullins et al. 1996. Jaw and branchial arch mutants in zebrafish I: Branchial arches. Development 123: 329-344.

Schlunegger, M.P. and M.G. Grütter. 1992. An unusual feature revealed by the crystal structure at 2.2 A resolution of human transforming growth factor- $\beta 2$. Nature 358: 430-434.

Schulte-Merker, S., R.K. Ho, B.G. Hermann, and C. Nüsslein-Volhard. 1992. The protein product of the zebrafish homologue of the mouse $\mathrm{T}$ gene is expressed in nuclei of the germ ring and the notochord of the early embryo. Development 116: 1021-1032.

Sepich, D.S., J. Wegner, S. O'Shea, and M. Westerfield. 1998. An altered intron inhibits synthesis of the acetylcholine receptor alpha-subunit in the paralyzed zebrafish mutant nic1. Genetics 148: $361-372$.

Sidow, A. 1996. Gen(om)e duplications in the evolution of early vertebrates. Curr. Opin. Genet. Dev. 6: 715-722.

Spaniol, P., C. Bornmann, G. Hauptmann, and T. Gester. 1996. Class III POU genes of zebrafish are predominantly expressed in the central nervous system. Nucleic Acid Res. 24: 4874-4881.

Stock, D.W., D.L. Ellies, Z. Zhao, M. Ekker, F.H. Ruddle, and K.M. Weiss. 1996. The evolution of the vertebrate Dlx gene family. Proc. Natl. Acad. Sci. 93: 10858-10863.

Storm, E.E. and D.M. Kingsley. 1996. Joint patterning defects caused by single and double mutations in members of the bone morphogenetic protein (BMP) family. Development 122: 3969-3979.

Storm, E.E., T.V. Huynh, N.G. Copeland, N.A. Jenkins, D.M. Kingsley, and S.J. Lee. 1994. Limb alterations in brachypodism mice due to mutations in a new member of the TGF $\beta$-superfamily. Nature 368: 639-643.

Sumiyama, K., K. Washio-Watanabe, T. Ono, M.C. Yoshida, T. Hakakawa, and S. Ueda. 1998. Human class III POU genes, POU3F1 and POU3F3, map to chromosomes 1p34.1 and 3p14.2. Mamm. Genome 9: 180-181.

Thomas, J.T., K. Lin, M. Nandedkar, M. Camargo, J. Cervenka, and F.P. Luyten. 1996. A human chondrodysplasia due to a mutation in a TGF- $\beta$ superfamily member. Nature Genet. 12: 315-317.

Westerfield, M. 1994. The zebrafish book: A guide for the laboratory use of zebrafish (Brachydanio rerio). University of Oregon Press, Eugene, OR.

Wolfman, N.M., G. Hattersley, K. Cox, A.J. Celeste, R. Nelson, N. Yamaji, J.L. Dube, E. DiBlasio-Smith, J. Nove, J.J. Song, J.M. Wozney, and V. Rosen. 1997. Ectopic induction of tendon and ligament in rats by growth and differentiation factors 5,6 , and 7 , members of the TGF- $\beta$ gene family. J. Clin. Invest. 100: $321-330$.

Yamashita, H., A. Shimizu, M. Kato, H. Nishitoh, H. Ichijo, A. Hanyu, I. Morita, M. Kimura, F. Makishima, and K. Miyazono. 1997. Growth/differentiation factor-5 induces angiogenesis in vivo. Exp. Cell Res. 235: 218-226.

Zardoya, R., E. Abouheif, and A. Meyer. 1996. Evolution and orthology of hedgehog genes. Trends Genet. 12: 496-497.

Received August 26, 1998; accepted in revised form December 7, 1998. 


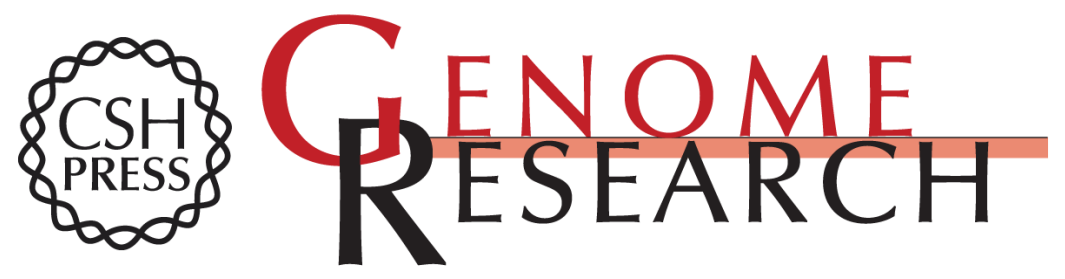

\section{Isolation of Zebrafish gdf7 and Comparative Genetic Mapping of Genes Belonging to the Growth/Differentiation Factor 5, 6, 7 Subgroup of the TGF- $\beta$ Superfamily}

Alan J. Davidson, John H. Postlethwait, Yi-Lin Yan, et al.

Genome Res. 1999 9: 121-129

Access the most recent version at doi:10.1101/gr.9.2.121

References This article cites 43 articles, 20 of which can be accessed free at: http://genome.cshlp.org/content/9/2/121.full.html\#ref-list-1

License

Email Alerting

Receive free email alerts when new articles cite this article - sign up in the box at the Service top right corner of the article or click here.

\section{Affordable, Accurate Sequencing.}

